OPEN ACCESS

Edited by:

Yancheng Li,

University of Technology Sydney,

Australia

Reviewed by:

Wanli Song

Northeastern University, China

Wenling Zhang,

Nanjing University of Science

and Technology, China

${ }^{*}$ Correspondence:

Xufeng Dong

dongxf@dlut.edu.cn

Min Qi

minqi@dlut.edu.cn

Specialty section:

This article was submitted to

Smart Materials,

a section of the journal

Frontiers in Materials

Received: 28 January 2021

Accepted: 25 February 2021

Published: 29 March 2021

Citation:

Tong Y, Li X, Zhao P, Dong X

Wu Z and Qi M (2021) Improved Magnetorheological Properties by Using lonic Liquid as Carrier Liquid

of Magnetorheological Fluids.

Front. Mater. 8:659998.

doi: 10.3389/fmats.2021.659998

\section{Improved Magnetorheological Properties by Using lonic Liquid as Carrier Liquid of Magnetorheological Fluids}

\author{
Yu Tong ${ }^{1}$, Xiaoguang Li", Penghui Zhao', Xufeng Dong ${ }^{1 *}$, Zhanjun $W u^{2}$ and Min $\mathrm{Qi}^{1 *}$ \\ ${ }^{1}$ School of Materials Science and Engineering, Dalian University of Technology, Dalian, China, ${ }^{2}$ School of Aeronautics \\ and Astronautics, Dalian University of Technology, Dalian, China
}

The interface between the particles and the carrier fluids has an important influence on the performance of magnetorheological fluid (MRF). In this study, ionic liquids and silicone oils with the same viscosity and different surface tensions were used as carrier fluids to prepare two different carbonyl iron powder (CIP) magnetorheological fluids. The rheological properties of the two magnetorheological fluids were evaluated by the MCR301 rotating rheometer. The experimental results indicate that ionic liquid-based MRF showed higher shear yield strength and more significant MR effect than silicone oil-based ones in higher magnetic field strength. A possible explanation was proposed and proved through experimental data analysis.

Keywords: magnetorheological fluids, ionic liquid, interface, MR effect, shear stress

\section{INTRODUCTION}

Magnetorheological fluid (MRF) is an intelligent suspension that responds to a magnetic field (de Vicente et al., 2011; Xu et al., 2011; Ahamed et al., 2018). Its special feature is that under an external magnetic field, the originally flowing liquid can be transformed into a solid-like state, and when the magnetic field is removed, the original flow state can be restored (Carlson and Jolly, 2000). Such characteristics make MRF have broad application prospects in various fields such as smart dampers, magnetic control clutches, and drug delivery (Olabi and Grunwald, 2007; Harris, 2011; Oh et al., 2013; Han et al., 2014; Najmaei, 2014; Phu and Choi, 2019). MRF is mainly composed of soft magnetic particles and non-magnetic carrier fluid. The most mature one is the combination of silicone oil and BASF's carbonyl iron powder (CIP) (Carlson et al., 1996). Improving the shear yield strength of MRF is an important research direction for its application. Although many studies in recent years have shown that special particle morphology can improve the overall performance of MRF (Tong et al., 2017; Xia et al., 2017; Wang et al., 2019; Han et al., 2020), due to the cheap price and high saturation magnetization, CIP is still the mainstay in actual use.

The MR effect is mainly attributed to the field-induced magnetization of the dispersing particles. When a magnetic field is applied, the magnetized particles attract one anther along the field direction and form chains or even columns (Jolly et al., 1996; Morillas and de Vicente, 2019). 
As a result, the suspension cannot flow until the applying shear stress reaches a critical value, which is defined as the yield stress. Increasing the shear yield strength of MRF from the carrier fluid has also been studied (Bossis et al., 2002; de Vicente et al., 2011; Ashtiani et al., 2015). Although the performance of MRF can be improved by using some special carrier fluids, the mechanism is still not very clear and cannot guide the development of new MRF with excellent performance.

Through previous research, it is found that the interface between the magnetic particle and the carrier liquid is an important factor affecting its performance. The performance of MRF can be improved by improving the two-phase interface. The traditional method of using surfactants to change the interface will complicate the composition of the rheological fluid, and cannot distinguish its influencing factors and effects well. Although ionic liquids have been used as carrier fluids for MRF (Guerrero-Sanchez et al., 2007; Gómez-Ramírez et al., 2011; Jönkkäri et al., 2014; Bombard et al., 2015), few people have noticed that although its viscosity is similar to that of silicone oil, its surface tension is higher than that of silicone oil. This will bring different interface effects. In this article, we will use ionic liquid as the carrier fluid of MRF to test the performance of ionic liquid-based MRF, and analyze the influence of interfacial on MRF performance by comparing with silicone oil-based MRF.

\section{EXPERIMENTAL}

\section{Preparation of MR Fluid}

MR fluids were prepared through a typical preparation process (Tong et al., 2017). Silicone oil (bought from Beijing Hangping silicon and chemical Co., Ltd., $0.5 \mathrm{~Pa} \cdot \mathrm{s}$ ) and 1-octyl-3methylimidazole tetrafluoroborate (ionic liquid, bought from Linzhou Keneng Material Technology Co., Ltd., 0.44 Pa.s) were used as the carrier liquid. The value of surface tension of silicone oil is $15.9-21 \mathrm{mN} / \mathrm{m}$ and that of ionic liquid is $25-45 \mathrm{mN} / \mathrm{m}$. Commercial CN carbonyl iron powder (BASF) was selected as the dispersed phase. They were named as SO-MRF and IL-MRF for short in the following text. The concentration of the two MR fluids were $20 \%$ in volume function. All reagents were of analytical grade and used without further purification.

\section{Evaluation of MR Fluids}

The magnetorheological properties of MR fluids were measured by a Physica MCR301 rheometer (Anton Paar, Austria) equipped with a magnetorheological module (MRD180). The rheometer can apply different magnetic fields on the parallel plate system (PP20) by changing the direct current. The test gap was 1 $\mathrm{mm}$. The shear yield strength and viscosity of MRF were tested in steady-state shear mode. Tested the curve of shear stress increasing linearly with shear rate from 10 to $100 \mathrm{~s}^{-1}$ under different magnetic fields. The response curve of MRF with magnetic field changes at a shear rate of $1 \mathrm{~s}^{-1}$ was tested. The variation curves of MRF modulus with shear strain amplitude (0.01-100\%) and applied magnetic field $\left(0-436 \mathrm{kA} \cdot \mathrm{m}^{-1}\right)$ were tested through the oscillating shear mode. All the tests were taken at $25^{\circ} \mathrm{C}$.

\section{RESULTS AND DISCUSSION}

The shear yield strength of MRF is an important criterion for evaluating its performance. For MRF, the high shear yield strength reflects its higher MR effect, which is very beneficial for the application of MRF. Figure $\mathbf{1}$ is the curve of the shear yield strength of two MRFs as a function of the magnetic field strength (obtained by fitting the results in Supplementary Figure S1 with Bingham model). The shear yield strength of the two MRFs in the figure increased with the increase of the test magnetic field strength, showing a typical MR effect. It can be clearly seen from the figure that IL-MRF has a higher shear yield strength, which shows that it has a higher MR effect. It is particularly worth noting that under higher magnetic field strength, the difference in shear yield strength of the two MRFs is further enlarged. Because the same CIP was used as the dispersed phase, the only difference in

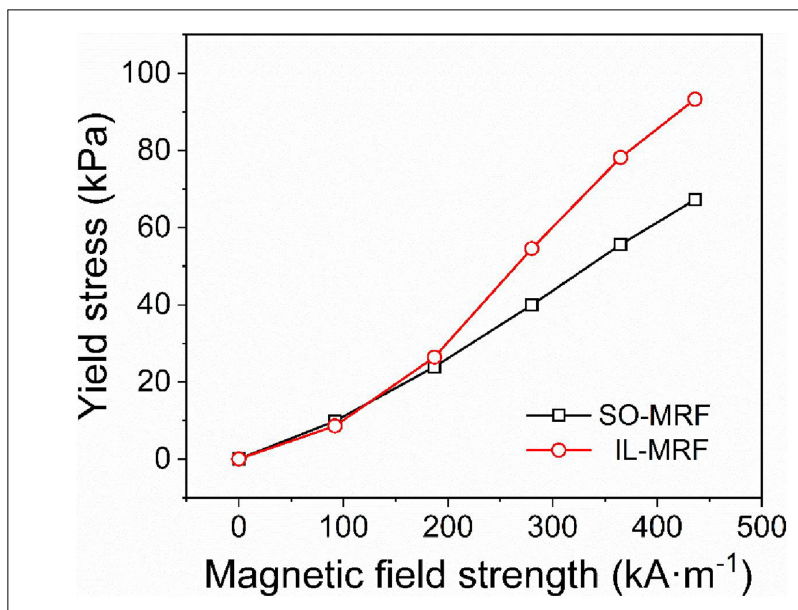

FIGURE 1 | Dependence of yield stress of SO-MRF and IL-MRF on magnetic field.

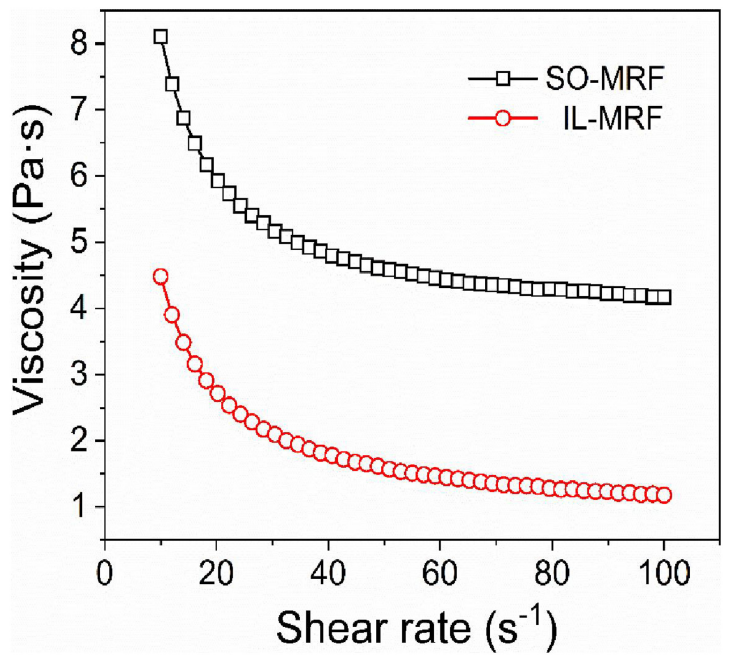

FIGURE 2 | Dependence of viscosity of SO-MRF and IL-MRF on shear rate at zero field. 


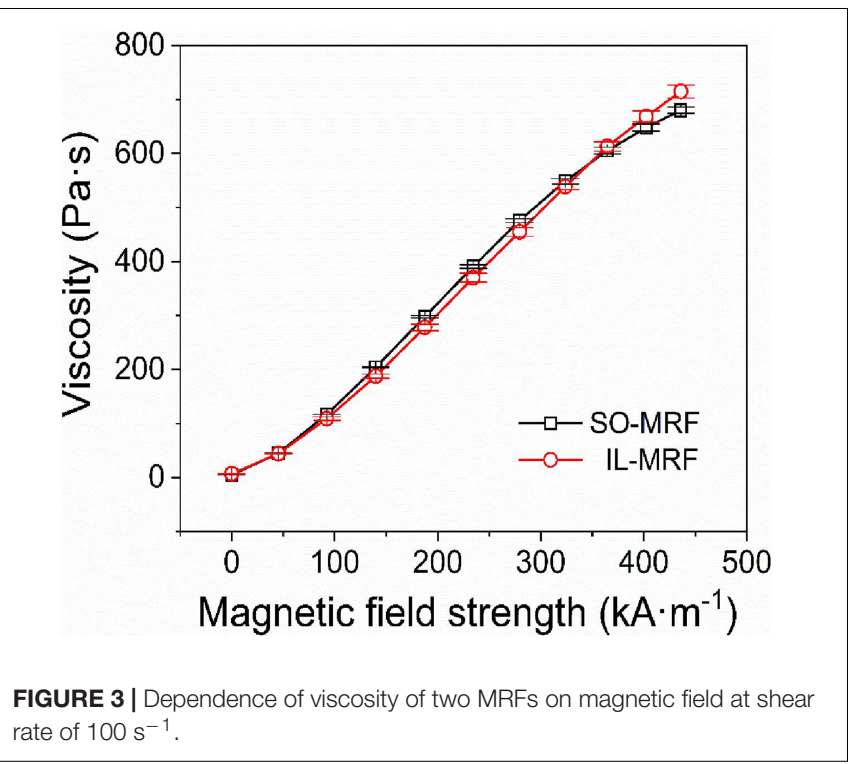

MRF was the carrier liquid. Then the performance improvement of IL-MRF has an important relationship with the ionic liquid. The reasons for the improved performance will be analyzed by studying the viscosity of the two MRFs.

Figure 2 is the dependence of viscosity of SO-MRF and IL-MRF on shear rate at zero field. The viscosity of the two magnetorheological fluids decreased as the shear rate increased, showing a typical shear thinning effect. In the zero field, it can be seen that the viscosity of SO-MRF is higher than that of IL-MRF. This is because the viscosity of silicone oil is higher than that of ionic liquid (Supplementary Figure S2). Supplementary Figure S3 shows the viscosity of this two MRFs under different magnetic field strength. Considering that their values are relatively close, the relationship between the viscosity and the magnetic field strength at a shear rate of $100 \mathrm{~s}^{-1}$ (When the shear rate is greater than $100 \mathrm{~s}^{-1}$, the viscosity is relatively stable) was tested, as shown in Figure 3. In Figure 3, the viscosity

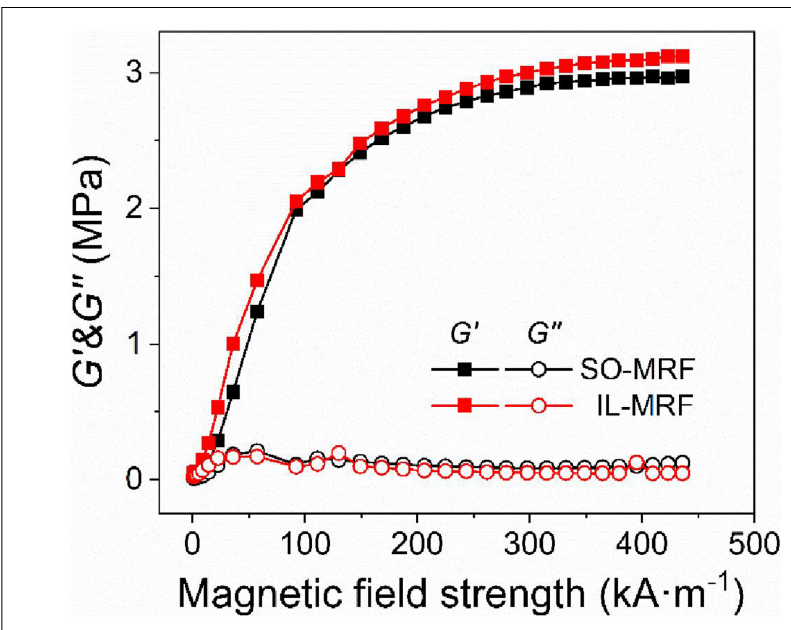

FIGURE 5 | Dependence of the storage modulus $G^{\prime}$ and loss modulus $G^{\prime \prime}$ of SO-MRF and IL-MRF on magnetic field at $0.01 \%$ stain amplitude and 10 $\mathrm{rad} \cdot \mathrm{s}^{-1}$ angular frequency. of the two MRFs increased with the increase of the magnetic field strength, showing a typical MR effect. In a lower magnetic field, since the viscosity of silicone oil is higher than that of ionic liquid, the viscosity of SO-MRF is slightly higher than that of IL-MRF. However, as the magnetic field strength increased, the viscosity increase rate of IL-MRF is higher than that of SO-MRF. Under a higher magnetic field, the viscosity of IL-MRF is higher than that of SO-MRF. This interesting phenomenon is inseparable from the higher surface tension of ionic liquids (Wasserscheid and Keim, 2000). Unlike the larger molecules of silicone oil, there are small ion fragments in ionic liquids, and its larger surface tension is related to this special ion fragment. We believe that these ion fragments are small relative to the particle size of CIP, so they are easily adsorbed on the surface of CIP to form an ion layer, like showing in Figure 4. This ion layer approaches each other when the particles are approached by the magnetic field. The van der Waals force between them will enhance the interaction

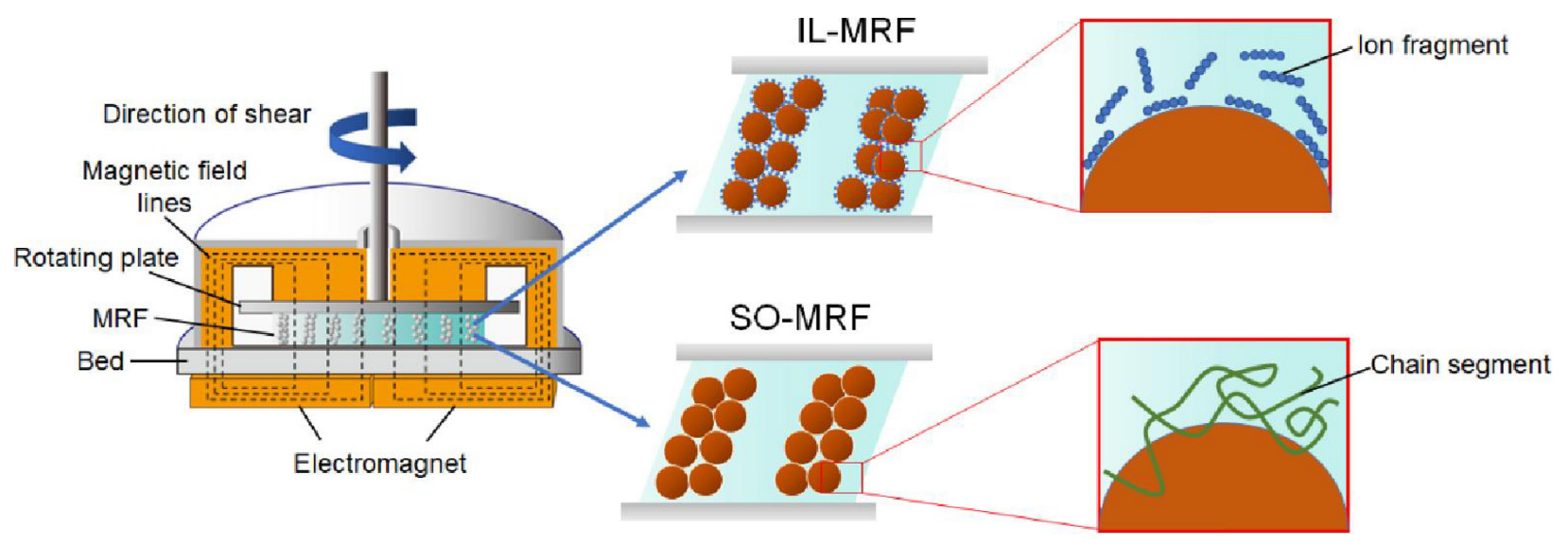

FIGURE 4 | Imaginary schematic diagram of the interface of SO-MRF and IL-MRF. 
between the particles, thus forming a more stable structure, which ultimately makes IL-MRF have a higher shear yield strength and higher viscosity. This enhancement effect is amplified as the distance between particles decreases. However, it is difficult to directly observe this layer of ion fragments. Therefore, we indirectly prove our guess by analyzing the structural stability of the two MRFs.

The modulus of MRF can reflect the stability of its structure. From Supplementary Figure S4, we determined the linear viscoelastic region of the two MRFs, and selected the strain amplitude of $0.01 \%$ and the angular frequency of $10 \mathrm{rad} \cdot \mathrm{s}^{-1}$ as the test conditions to test the dependence of the modulus of SO-MRF and IL-MRF on different magnetic fields. The results in Figure 5 show that the storage modulus $G^{\prime}$ of IL-MRF is higher than that of SO-MRF, and its loss modulus $G^{\prime \prime}$ is lower than that of SO-MRF, which indicates that the structure formed by CIP in IL-MRF is more stable. As other conditions are the same, this proves our previous guess that the special properties of ionic liquids enhance the interaction between particles to improve the performance of MRF. Of course, to prove this conjecture, more experiments, such as computational simulations, are needed.

\section{CONCLUSION}

In this research, an ionic liquid-based magnetorheological fluid was produced and its performance was analyzed through testing. Experimental results showed that the shear yield strength of IL-MRF was significantly improved in higher magnetic field strength. The ion fragments in the ionic liquid would form an ion layer on the surface of the particles to improve the interaction between the particles and enhance the MR effect of IL-MRF under

\section{REFERENCES}

Ahamed, R., Choi, S., and Ferdaus, M. M. (2018). A state of art on magnetorheological materials and their potential applications. J. Intell. Mater. Syst. Struct. 29, 2051-2095. doi: 10.1177/1045389X18754350

Ashtiani, M., Hashemabadi, S. H., and Ghaffari, A. (2015). A review on the magnetorheological fluid preparation and stabilization. J. Magn. Magn. Mater. 374, 716-730. doi: 10.1016/j.jmmm.2014.09.020

Bombard, A. J. F., Gonçalves, F. R., and de Vicente, J. (2015). Magnetorheology of carbonyl iron dispersions in 1-Alkyl-3-methylimidazolium ionic liquids. Ind. Eng. Chem. Res. 54, 9956-9963. doi: 10.1021/acs.iecr.5b02824

Bossis, G., Volkova, O., Lacis, S., and Meunier, A. (2002). "Magnetorheology: fluids, structures and rheology," in Ferrofluids: Magnetically Controllable Fluids and Their Applications, ed. S. Odenbach (Berlin: Springer Berlin Heidelberg), 202-230. doi: 10.1007/3-540-45646-5_11

Carlson, J. D., Catanzarite, D. M., and St Clair, K. A. (1996). Commercial magnetorheological fluid devices. Int. J. Mod. Phys. B 10, 2857-2865. doi: 10.1142/ S0217979296001306

Carlson, J. D., and Jolly, M. R. (2000). MR fluid, foam and elastomer devices. Mechatronics 10, 555-569. doi: 10.1016/S0957-4158(99)00064-1

de Vicente, J., Klingenberg, D. J., and Hidalgo-Alvarez, R. (2011). Magnetorheological fluids: a review. Soft Matter 7, 3701-3710. doi: 10.1039/ COSM01221A

Gómez-Ramírez, A., López-López, M. T., González-Caballero, F., and Durán, J. D. G. (2011). Stability of magnetorheological fluids in ionic liquids. Smart Mater. Struct. 20:045001. doi: 10.1088/0964-1726/20/4/045001 a magnetic field. This enhancement effect is confirmed by the MRF modulus results. The experimental results can explain our hypothesis, but more precise analysis and calculations will be reflected in our future work.

\section{DATA AVAILABILITY STATEMENT}

All datasets generated for this study are included in the article/Supplementary Material, further inquiries can be directed to the corresponding author/s.

\section{AUTHOR CONTRIBUTIONS}

YT: methodology and writing-original draft preparation. $\mathrm{XL}$ : preparation and test. PZ: mechanism. XD: supervision, conceptualization, and writing-reviewing and editing. ZW: visualization. MQ: validation. All authors contributed to the article and approved the submitted version.

\section{FUNDING}

This research was financial funded by the National Key R\&D Program of China under the Grant No. 2018YFC0705603.

\section{SUPPLEMENTARY MATERIAL}

The Supplementary Material for this article can be found online at: https://www.frontiersin.org/articles/10.3389/fmats. 2021.659998/full\#supplementary-material

Guerrero-Sanchez, C., Lara-Ceniceros, T., Jimenez-Regalado, E., Raşa, M., and Schubert, U. S. (2007). Magnetorheological fluids based on ionic liquids. $A d v$. Mater. 19, 1740-1747. doi: 10.1002/adma.200700302

Han, W. J., An, J. S., and Choi, H. J. (2020). Enhanced magnetorheological characteristics of hollow magnetite nanoparticle-carbonyl iron microsphere suspension. Smart Mater. Struct. 29:055022. doi: 10.1088/1361-665x/ab7f43

Han, Y., Oh, J., Kim, J., and Choi, S. (2014). Design and experimental evaluation of a tactile display featuring magnetorheological fluids. Smart Mater. Struct. 23:077001. doi: 10.1088/0964-1726/23/7/077001

Harris, D. C. (2011). "History of magnetorheological finishing," in Proceedings of the SPIE (Bellingham, WA: SPIE) 8016, 1-22.

Jolly, M. R., Carlson, J. D., and Muñoz, B. C. (1996). A model of the behaviour of magnetorheological materials. Smart Mater. Struct. 5, 607-614. doi: 10.1088/ 0964-1726/5/5/009

Jönkkäri, I., Isakov, M., and Syrjälä, S. (2014). Sedimentation stability and rheological properties of ionic liquid-based bidisperse magnetorheological fluids. J. Intell. Mater. Syst. Struct. 26, 2256-2265. doi: 10.1177/1045389X 14551436

Morillas, J. R., and de Vicente, J. (2019). Yielding behavior of model magnetorheological fluids. Soft Matter 15, 3330-3342. doi: 10.1039/C9SM0 $0275 \mathrm{H}$

Najmaei, N. (2014). Design of a Haptic Interface for Medical Applications Using Magneto-Rheological Fluid Based Actuators. London, UK: The University of Western Ontario.

Oh, J. S., Kim, J. K., Lee, S. R., Choi, S. B., and Song, B. K. (2013). Design of tactile device for medical application using magnetorheological 
fluid. J. Phys. Conf. Ser. 412:012047. doi: 10.1088/1742-6596/412/1/ 012047

Olabi, A. G., and Grunwald, A. (2007). Design and application of magnetorheological fluid. Mater. Des. 28, 2658-2664. doi: 10.1016/j.matdes.2006. 10.009

Phu, D. X., and Choi, S. (2019). Magnetorheological fluid based devices reported in 2013-2018: mini-review and comment on structural configurations. Front. Mater. 6:19. doi: 10.3389/fmats.2019.00019

Tong, Y., Dong, X., and Qi, M. (2017). High performance magnetorheological fluids with flower-like cobalt particles. Smart Mater. Struct. 26:025023. doi: 10.1088/1361-665X/aa57cc

Wang, G., Zhao, D., Li, N., Zeng, Y., Han, S., Ma, Y., et al. (2019). Facile synthesis of hierarchically structured flower-like Fe3O4 microspheres for high-performance magnetorheological fluids. J. Ind. Eng. Chem. 79, 217-225. doi: 10.1016/j.jiec. 2019.06.040

Wasserscheid, P., and Keim, W. (2000). Ionic liquids-new "solutions" for transition metal catalysis. Angew. Chem. Int. Ed. 39, 3772-3789. doi: 10.1002/1521-3773(20001103)39:21<3772::AID-ANIE3772<3.0.CO;2-5
Xia, Z., Wu, X., Peng, G., Wang, L., Li, W., and Wen, W. (2017). A novel nickel nanowire based magnetorheological material. Smart Mater. Struct. 26:054006. doi: 10.1088/1361-665x/aa5bd0

Xu, Y., Gong, X., Xuan, S., Zhang, W., and Fan, Y. (2011). A highperformance magnetorheological material: preparation, characterization and magnetic-mechanic coupling properties. Soft Matter 7:5246. doi: 10.1039/c1sm $05301 \mathrm{a}$

Conflict of Interest: The authors declare that the research was conducted in the absence of any commercial or financial relationships that could be construed as a potential conflict of interest.

Copyright (C) 2021 Tong, Li, Zhao, Dong, Wu and Qi. This is an open-access article distributed under the terms of the Creative Commons Attribution License (CC BY). The use, distribution or reproduction in other forums is permitted, provided the original author(s) and the copyright owner(s) are credited and that the original publication in this journal is cited, in accordance with accepted academic practice. No use, distribution or reproduction is permitted which does not comply with these terms. 